\title{
LA-UR-20-22781
}

Approved for public release; distribution is unlimited.

Title:

Author(s):

Issued:
w19_OMEC Scientific Highlight

Zelenay, Piotr

Yin, $X i$

Holby, Edward F.

Intended for: Web

2020-04-07 
Disclaimer:

Los Alamos National Laboratory, an affirmative action/equal opportunity employer, is operated by Triad National Security, LLC for the National Nuclear Security Administration of U.S. Department of Energy under contract 89233218CNA000001. By approving this article, the publisher recognizes that the U.S. Government retains nonexclusive, royalty-free license to publish or reproduce the published form of this contribution, or to allow others to do so, for U.S. Government purposes. Los Alamos National Laboratory requests that the publisher identify this article as work performed under the auspices of the U.S. Department of Energy. Los Alamos National Laboratory strongly supports academic freedom and a researcher's right to publish; as an institution, however, the Laboratory does not endorse the viewpoint of a publication or guarantee its technical correctness. 


\section{w19_OMEC Scientific Highlight}

\section{Scientific Achievement}

$\square$ Revealed the effect of graphite substrate on $\mathrm{H}$ binding / hydrogen evolution reaction (HER) activity of organic molecular electrocatalysts (OMECs)

$\square$ Demonstrated the effect of molecular modification on calculated HER activity descriptor

\section{Significance and Impact}

$\square$ Understanding of HER processes is important for discovering low-cost OMECs for hydrogen production and energy application

$\square$ Design of OMEC structures remains challenging due to the unexplored redox properties of organic molecules

$\square$ Density functional theory (DFT) has been proposed to provide needed insights into HER activity for improved materials design

\section{Research Details}

$\square$ DFT calculations on the binding energy of HER intermediates are used for predicting reduction potentials and calculated $\mathrm{H}$ adsorption energy as activity descriptor

- LANL Institutional Computing (IC) resources are essential for successful project execution given the size of structures involved in modeling molecule-support interaction

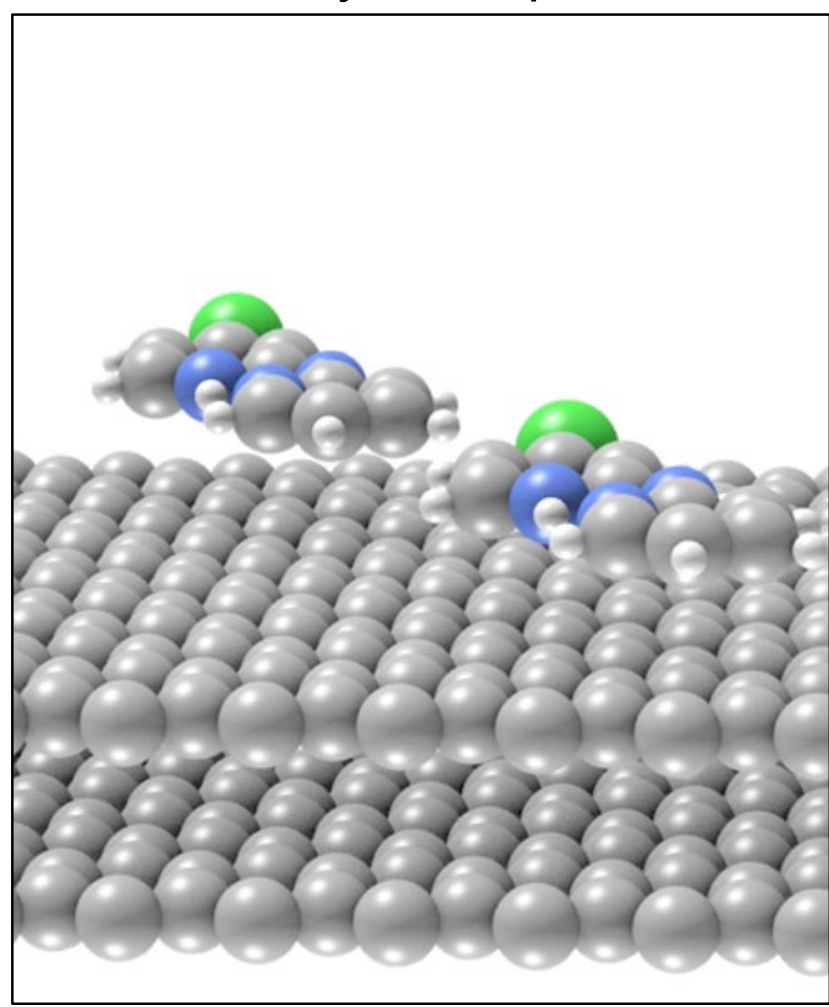

Adsorption of $\mathrm{H}$ atom at pyridyl- $\mathrm{N}$ site in chlorine-substituted DPA molecule on graphite support surface. 